\section{Desarrollo de vacunas biotecnológicas en Cuba y Argentina}

\section{Ciencia Tecnología y Política \\ Año 4 N 7 Noviembre 2021}

\begin{abstract}
Resumen: La pandemia causada por la Covid-19 pone de manifiesto descarnadamente que el desarrollo de vacunas biotecnológicas representa una función social clave para los sistemas de salud de los países y un aspecto central de soberanía sanitaria. En este artículo se analizan las políticas públicas implementadas por Cuba y Argentina en el periodo 1980-2020 asociadas con el desarrollo de vacunas biotecnológicas. Se muestra que Cuba se convirtió en un país líder al seguir una política de ciclo cerrado permitiéndole crear una plataforma científico-tecnológica virtuosa, orientada a cubrir las necesidades sanitarias de su población, controlando el diseño, patentamiento, producción y comercialización de vacunas. Por su parte, en Argentina se han creado planes, programas y estrategias puntuales de impulso y financiamiento al sector biotecnológico en general, y al diseño de vacunas en particular, permitiendo la consolidación de la ciencia básica asociada a este campo. Sin embargo, no se ha logrado vincular la I+D con la producción y comercialización, tampoco con la resolución de las necesidades y demandas sociales en materia de salud de su población.
\end{abstract}

Palabras clave: biotecnología, vacunas biotecnológicas, desarrollo, Cuba, Argentina.

\section{Development of biotechnological vaccines in Cuba and Argentina}

\begin{abstract}
The pandemic caused by Covid-19 clearly shows that the development of biotechnological vaccines represents a key social function for the health systems of countries and a central aspect of health sovereignty. This article analyzes the public policies implemented by Cuba and Argentina during the period of 1980 and 2020 associated with the development of biotechnological vaccines. It is shown that Cuba became a leading country by following a closed-loop policy, allowing it to create a virtuous scientific-technological platform, aimed at meeting the health needs of its population, controlling the design, patenting, producing and marketing of vaccines. On the other hand, in Argentina, specific plans, programs and strategies have been created to promote and finance the biotechnology sector in general, and the design of vaccines in particular, allowing the consolidation of the basic science associated with this field. However, it has not been possible to link R\&D with production and commercialization, neither with the resolution of the social needs and demands in terms of health of its population.
\end{abstract}

Keywords: biotechnology, biotechnological vaccines, development, Cuba, Argentina.

\section{Desenvolvimento de vacinas biotecnológicas em Cuba e na Argentina}

Resumo: A pandemia causada pela Covid-19 mostra claramente que o desenvolvimento de vacinas biotecnológicas representa uma função social chave para os sistemas de saúde dos países e um aspecto central da soberania da saúde. Este artigo analisa as políticas públicas implementadas por Cuba e pela Argentina no período 1980-2020 associadas ao desenvolvimento de vacinas biotecnológicas. Mostra-se que Cuba se tornou um país líder ao seguir uma política de ciclo fechado que lhe permitiu criar uma virtuosa plataforma científico-tecnológica voltada para atender às necessidades de saúde de sua população, controlando o design, o patenteamento, a produção e a comercialização de vacinas. Por outro lado, na Argentina foram elaborados planos, programas e estratégias específicas para promover e financiar o setor de biotecnologia em geral e o desenho de vacinas em particular, permitindo a consolidação da ciência básica associada a esse campo. Porém, não tem sido possível vincular a P\&D à produção e comercialização, nem à resolução das necessidades e demandas sociais em termos de saúde de sua população.

Palavras-chave: biotecnologia, vacinas biotecnológicas, desenvolvimento, Cuba, Argentina.
Nancy Alejandra Cuevas

Mercado

Doctora en Estudios del Desarrollo Universidad Autónoma de Zacatecas (México)

nancycuevas232615@gmail.com

Mónica Guadalupe Chávez

Elorza

Doctora en Política Pública Universidad Autónoma de Zacatecas (México) monick.elorza@uaz.edu.mx

Año $4 \mathrm{~N}^{\circ} 7$ Noviembre 2021 Fecha de recibido: 29/07/2021 Fecha de aprobado: 12/10/2021 https://doi.org/10.24215/26183188e065 https://revistas.un|p.edu.ar/CTyP ISSN 2618-3188

\section{ISSN 2618-3188}

(




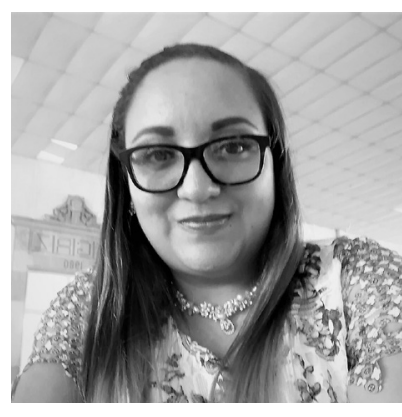

Nancy Alejandra Cuevas Mercado

Doctora en Estudios del Desarrollo

Universidad Autónoma de Zacatecas (México)

nancycuevas232615@gmail. com

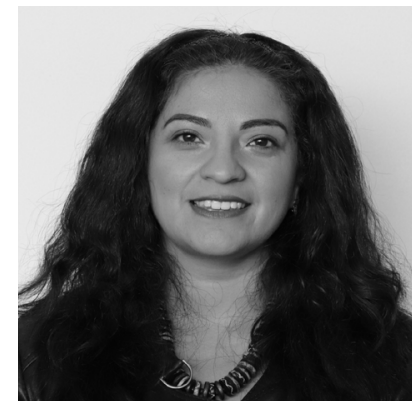

Mónica Guadalupe Chávez Elorza

Doctora en Política Pública

Universidad Autónoma de

Zacatecas (México)

monick.elorza@uaz.edu.mx

\section{Desarrollo de vacunas biotecnológicas en Cuba y Argentina}

Resumen: La pandemia causada por la Covid-19 pone de manifiesto descarnadamente que el desarrollo de vacunas biotecnológicas representa una función social clave para los sistemas de salud de los países y un aspecto central de soberanía sanitaria. En este artículo se analizan las políticas públicas implementadas por Cuba y Argentina en el periodo 1980-2020 asociadas con el desarrollo de vacunas biotecnológicas. Se muestra que Cuba se convirtió en un país líder al seguir una política de ciclo cerrado permitiéndole crear una plataforma científicotecnológica virtuosa, orientada a cubrir las necesidades sanitarias de su población, controlando el diseño, patentamiento, producción y comercialización de vacunas. Por su parte, en Argentina se han creado planes, programas y estrategias puntuales de impulso y financiamiento al sector biotecnológico en general, y al diseño de vacunas en particular, permitiendo la consolidación de la ciencia básica asociada a este campo. Sin embargo, no se ha logrado vincular la I+D con la producción y comercialización, tampoco con la resolución de las necesidades y demandas sociales en materia de salud de su población.

\section{Introducción}

Entre 1960 y 1970 surgió en América Latina el Pensamiento Latinoamericano en Ciencia Tecnología y Desarrollo (PLACTED), movimiento intelectual orientado a buscar soluciones a los problemas del crecimiento económico e impulsar la modernización social dando un papel destacado a la ciencia y la tecnología (CyT). Posteriormente, las políticas de CyT han cambiado de estilo y enfoque, siguiendo las tendencias prevalecientes en la mayor parte del mundo, sin abandonar la idea central del desarrollo. El foco está centrado ahora -muchas veces en forma más discursiva que real- en los procesos de innovación y la difusión social de conocimientos (Albornoz, 2009).

En la búsqueda por alcanzar progreso y bienestar social, los principales diseñadores de políticas públicas realizan recomendaciones sobre áreas estratégicas que se deben impulsar. La CyT es considerada trascendental por su potencial de incentivar el crecimiento económico y el bienestar de la población. La biotecnología moderna cobra importancia al ser una actividad multidisciplinaria con la capacidad de solucionar problemas (Bolívar 
| Ciencia, Tecnología y Política | Año 4 | Nº7 | e065 | Noviembre 2021 | ISSN 2618-3188 | www.revistas.unlp.edu.ar/CTyP |

Zapata, 2001) en distintas áreas como la salud. En este marco, el desarrollo de vacunas biotecnológicas representa una función social clave para los sistemas de salud, por tratarse de una actividad científica importante en sí misma y por las implicaciones que tiene para la soberanía sanitaria.

El objetivo de este artículo es revisar la trayectoria y alcances de la política pública de impulso al sector biotecnológico en salud en Cuba y Argentina'. El marco teórico utilizado se basa en la propuesta de Varsavsky (1978) sobre estilo tecnológico o de desarrollo, es decir, el reto que enfrentan los países latinoamericanos de optar por emular las políticas CyT y agendas de investigación adoptadas por los países centrales, o bien, definir su propio rumbo tecnológico priorizando las necesidades de la población. Desde lo metodológico se realizó en primer lugar una revisión de los documentos oficiales y artículos sobre la política científica pública vinculada con el impulso a la biotecnología en ambos países, haciendo énfasis en el desarrollo de vacunas para uso humano. En segundo lugar, se realizó un análisis bibliométrico utilizando la plataforma Web of Science (WoS) para la búsqueda de publicaciones sobre vacunas para uso humano durante el periodo 1980 a 2020, asumiendo que las publicaciones son de algún modo una medida del desarrollo de la ciencia básica en el tema. Por último, se muestra el total de patentes concedidas tanto en Cuba como en Argentina en productos biotecnológicos y farmacéuticos utilizando la base de datos de la Organización Mundial de la Propiedad Intelectual (OMPI).

\section{Vacunas biotecnológicas en Cuba}

La trayectoria exitosa de la biotecnología en Cuba está relacionada con el estilo tecnológico adoptado a través de los años, dado que la meta no solo fue hacer un uso eficiente de los recursos, sino que, buscó solucionar problemas de salud de la población. En términos de Varsavsky (1978), Cuba eligió un plan nacional pueblocéntrico destinado a cubrir las necesidades de su población. Pero, paralelamente, ha llevado a cabo un plan empresocéntrico hacia el exterior. Una vez que fueron capaces de cubrir las necesidades internas, los centros de investigación y empresas comercializadoras siguieron las lógicas del mercado capitalista, vendiendo al exterior sus productos biotecnológicos, cumpliendo con las normativas internacionales de derechos de propiedad intelectual (DPI); configurándose como empresas competitivas a nivel mundial.

Gracias a las inversiones hechas por la Revolución Cubana en educación, ciencia y salud entre 1960 y 1970, Cuba educó a científicos e ingenieros, logrando contar con alrededor de 1,8 investigadores por cada 1.000 habitantes; en 1978 se inició un proyecto para producir interferones; y paralelamente, debido a los brotes de meningitis, dengue y conjuntivitis, aceleraron la decisión del gobierno de crear las primeras instituciones en el área de la biotecnología (López et al, 2006; Reid-Henry y Plahte, 2020; Plahte, 2010).

Entre 1990 y 1996, el gobierno invirtió cerca de 1 billón de dólares estadounidenses en la creación de polos científicos que operaban de manera integrada con instituciones de educación superior, empresas, instituciones de salud y los usuarios finales. El Polo científico del Oeste de la Habana, creado en 1992, operaba como estructura organizativa y coordinadora de la biotecnología cubana y estaba integrado por instituciones de investiga-

\footnotetext{
1 Este trabajo se realizó en el marco del Proyecto A1-S-9013 "Evaluación de impacto de las políticas públicas en la productividad científica, tecnológica e innovadora en México", Convocatoria de Investigación Básica 2017-2018 SEP-CONACYT.
} 
ción, educación, salud y economía. Agrupaba 38 instituciones pertenecientes a 13 organismos del Estado y alrededor de 12.000 trabajadores (más de 4.000 graduados universitarios, $12 \%$ de ellos investigadores) (OPS, 2020, párr.13).

El sector biotecnológico cubano se destaca principalmente porque: i) el Estado es el inversionista principal; ii) la biotecnología es parte del sistema de salud y se desarrollan productos para resolver los problemas sanitarios del país; iii) todos los productos son generados por científicos e investigadores locales y no por extranjeros; iv) la capacidad operativa es de "círculo cerrado"; significa que los centros biotecnológicos realizan el "ciclo completo" (investigación-desarrollo-producción-comercialización-seguimiento postventa); v) han mejorado la habilidad para acceder a mercados extranjeros, particularmente del mundo desarrollado, basado en calidad, volúmenes de producción, costos, novedad y empresas mixtas; vi) hay colaboración con otros países periféricos en la investigación, desarrollo, producción y comercialización de productos biotecnológicos (OPS, 2020; López et al, 2006).

Cuba creó una agencia reguladora de fármacos permitiéndole adoptar normas internacionales sobre biotecnología. Tal es el caso de las normas iso-9000, normas de buenas prácticas de fabricación, de laboratorio y clínicas. En cuestiones de propiedad intelectual, se adhirió en 1995 al Acuerdo sobre los Aspectos de Derechos de Propiedad Intelectual relacionados con el Comercio (ADPIC) y en 1996 firmó el Tratado de Cooperación en Materia de Patentes (PCT)². El Estado conservó la titularidad de la patente y otorgó el derecho de autoría a los inventores. Ello significa que el Es- tado define el rumbo de las invenciones logradas (Reid-Henry y Plahte, 2020: 223-224).

La industria biotecnológica hasta 2009 fue manejada por el Consejo de Estado y recibió un trato preferencial por parte del liderazgo político del país, asegurando el flujo de capital. Esta experiencia culminó con la creación de la Organización Superior del Desarrollo Empresarial (OSDE) y el Grupo de las Industrias Biotecnológicas y Farmacéuticas (BioCubaFarma) en noviembre de 2012, integrado por las entidades pertenecientes al Polo Científico y al Grupo Empresarial (QUIMEFA) (Consejo de Ministros, 2012; Cadalzo-Díaz et al., 2017; Reid-Henry y Plahte, 2020).

BioCubaFarma (2021) consta de 32 empresas, 65 unidades empresariales, 80 líneas de producción y 21 unidades científicas. Cuenta con más de 20.000 empleados (alrededor de 17.000 profesionales técnicos y operadores, 1.265 maestros y 279 doctores). Los centros y unidades científicas que lo componen fueron hasta 2016 responsables del $34,7 \%$ de la producción científica en biomedicina y del $16 \%$ del total de la producción biotecnológica en el país (Guzmán-Sánchez et al., 2018).

El viraje hacia BioCubaFarma guarda relación con la constitución de un andamiaje institucional y de infraestructura articulado con las necesidades de salud pública del país. Pero busca ser competitivo, como señalan Reid-Henry y Plahte (2020) en los últimos años durante el desarrollo de la vacuna EGF-r para el cáncer, Cuba ha negociado con laboratorios extranjeros para llevar a cabo las últimas fases que permitieran su aprobación. La realidad que impera requiere de colaboraciones internacionales; no obstante, el Estado no ha per-

\footnotetext{
2 No obstante, Cuba no suele compartir sus DPI con extranjeros; de acuerdo con Quach et al. (2006) el 95\% de las patentes biotecnológicas cubanas se concedieron a cesionarios cubanos.
} 
| Ciencia, Tecnología y Política | Año 4 | Nº | e065 | Noviembre 2021 | ISSN 2618-3188 | www.revistas.unlp.edu.ar/CTyP |

dido el control del sector biotecnológico.

\section{Vacunas biotecnológicas en Argentina}

La trayectoria de la biotecnología en salud en Argentina contrasta con la cubana. En principio, siguiendo a Varsavsky (1978), esta se ha instalado con características de índole "empresocéntrica" y no como una tecnología emergente enfocada a solucionar los problemas de la población. Se han destinado fondos públicos y se han promovido acuerdos sur-sur para la profesionalización de recursos humanos y el mejoramiento de transferencia de tecnología. Empero, no han seguido un ciclo completo, esto es, la mayoría de lo que se hace queda en el nivel de ciencia básica; sin mencionar que el objetivo principal está orientado al mercado, incluido el exterior y, sobre todo, no está articulado al sistema de salud argentino.

En Argentina, el Estado es el principal financiador de los desarrollos en biotecnología mediante subvenciones y créditos. El Ministerio de Ciencia, Tecnología e Innovación (MINCyT) es el encargado de impulsar la investigación, el desarrollo y la innovación a través del Consejo Nacional de Investigaciones Científicas y Técnicas (CONICET) y la Agencia Nacional para la Promoción de la Ciencia y la Tecnología (ANPCyT). Dos de los principales instrumentos de financiamiento son: el Fondo para la Investigación Científico-Tecnológica (FONCyT) y el Fondo Tecnológico Argentino (FONTAR) (Vitagliano y Villalpando, 2003: 29).

A su vez, la Administración de Nacional de Laboratorios e Institutos de Salud (ANLIS) vincula CyT con acciones sanitarias enfocadas a la prevención, diagnóstico, investigación y tratamiento de enfermedades; tiene responsabilidad en la producción y control de calidad de inmunobiológicos, ejecuta programas sanitarios, coordina las redes de laboratorios, realiza estudios epidemiológicos y capacita recursos humanos (Ministerio de Salud, 2021). Finalmente, el ente regulador de biotecnología en salud es la Comisión Nacional de Biotecnología y Salud (CONBYSA), que estudia y recomienda las normas vigentes que rigen el desarrollo, elaboración y aprobación de productos biotecnológicos destinados a la salud y consumo humano (Vitagliano y Villalpando, 2003: 43-44; MINCYT, 2010: 4).

Desde 1980 el Estado Nacional ejerció un rol importante en el desarrollo de la biotecnología impulsando diferentes programas: a) el Programa Nacional de Biotecnología de 1982-1991; b) el Programa Nacional Prioritario de Biotecnología (1992-1996), cuyos fondos se destinaron a proyectos de investigación concertados con el sector privado; c) el Programa de Biotecnología del Plan Plurianual de Ciencia y Tecnología (1998-2000), donde se establecieron prioridades temáticas de los proyectos de investigación y desarrollo; d) el Plan Nacional de Ciencia, Tecnología e Innovación 2004 que contempló tres instrumentos para impulsar la biotecnología: Centro Argentino-Brasileño de Biotecnología (CABBIO), Centro Internacional de Ingeniería Genética y Biotecnología (ICGEB), y programas y proyectos estratégicos en distintas áreas como la salud; e) el Plan Estratégico Nacional de Ciencia, Tecnología e Innovación "Bicentenario" (2006-2010), en el cual se definió a la biotecnología como área temática prioritaria; y f) el Plan Nacional de Ciencia, Tecnología e Innovación Productiva Argentina Innovadora 2020 en donde se estableció a la articulación de las tecnologías de propósito general (TPG), Biotecnología, Nanotecnología y TICs con sectores productivos de bienes y servicios para generar saltos cualitativos en términos de competitividad productiva y mejorar la calidad de vida (MINCyT y PEPP, s.f.: 41, 92, 100; MINCyT, 2010: 3; 
Vitagliano y Villalpando, 2003:28).

Se gestionaron varias iniciativas de cooperación con otras naciones, la creación del CABBIO en 1987 y la participación en actividades de investigación y formación avanzada con el ICGBEB de Naciones Unidas. En 1986 se creó el Foro Argentino de Biotecnología (FAB) para promover el área e impulsar la cooperación y colaboración entre los sectores científico, empresarial y gubernamental (MINCyT y SEPP, s.f.; Vitagliano y ViIlalpando, 2003; MINCyT, 2010: 4). Existen otros proyectos de cooperación con la Comisión Económica Europea (CEE) (Biotecsur, MERCOSUR, el Séptimo Programa Marco, el Programa Horizonte 2020³) (Anlló et al, 2016: 55) y el Centro Argentino-Cubano de Biotecnología Aplicada al Desarrollo de Vacunas y Fármacos (CACBVaF) creado en 2009 (López, 2019: 141).

En 2007, se promulgó la Ley de Promoción del Desarrollo y Producción de la Biotecnología Moderna (Ley No. 26.270/07) la cual busca promover el desarrollo y la producción de la biotecnología moderna en el país con vigencia de 15 años a partir de su promulgación (art.1) y crea el Fondo de Estímulo para el financiamiento de capital inicial en proyectos biotecnológicos (art. 15).

En Argentina los sectores en los que la biotecnología ha tenido más desarrollo han sido: agropecuario, alimentación, salud humana, diagnóstico, salud animal, algunos sectores industriales (químicos esencialmente) y el medio ambiente (Vitagliano y Villalpando, 2003). El MINCyT (2016: 10-11) realizó en 2015 la Encuesta Nacional de Empresas de Biotecnología encontrando que, de las 201 empresas encuestadas, 21\% se dedica al área de salud humana, seguida de reproducción animal (17\%), inoculantes (13\%), fertilización humana (11\%), insumos industriales $(10 \%)$, semillas $(9 \%)$, salud animal $(8 \%)$, micropropagación (6\%) y otros (5\%).

Es importante señalar las limitaciones y desafíos que tiene el sector en el país. Existen dificultades para el escalado y la transferencia de tecnología debido a que la mayor parte de las empresas tienen un tamaño insuficiente para realizarlas por su cuenta y no existen suficientes incentivos para que los investigadores y los centros prioricen estas actividades. Asimismo, se observa una escasa vinculación entre el complejo científico tecnológico y las empresas, si bien un gran porcentaje de los grupos de I+D muestran potencial de vinculación y transferencia con el sector productivo. La mayoría de los institutos de I+D no trabajan bajo normas internacionales de buenas prácticas, además que se percibe escasez, poca fluidez y falta de continuidad en el financiamiento en equipos y gastos operativos. Finalmente, en lo que respecta a los productos de salud humana, como es el caso de las vacunas, existe una gran concentración económica, condicionada por las economías de escala y masa crítica, esto es, no es rentable producir si no se vende de manera masiva (Anlló et al, 2016).

\section{Producción científica y patentes}

La producción científica refleja la orientación que toman las agendas de investigación de un lugar y un campo en particular. En este sentido, la producción científica relacionada con vacunas biotecnológicas, en el contexto actual de la pandemia por

\footnotetext{
${ }^{3}$ Horizonte 2020 es el programa de investigación e innovación más ambicioso puesto en marcha por la Unión Europea (UE) y se espera que dé lugar a más avances, descubrimientos y primicias a nivel mundial, convirtiendo las grandes ideas surgidas de los laboratorios en productos de mercado (Dirección General de Investigación e Innovación de la Comisión Europea, 2014).
} 


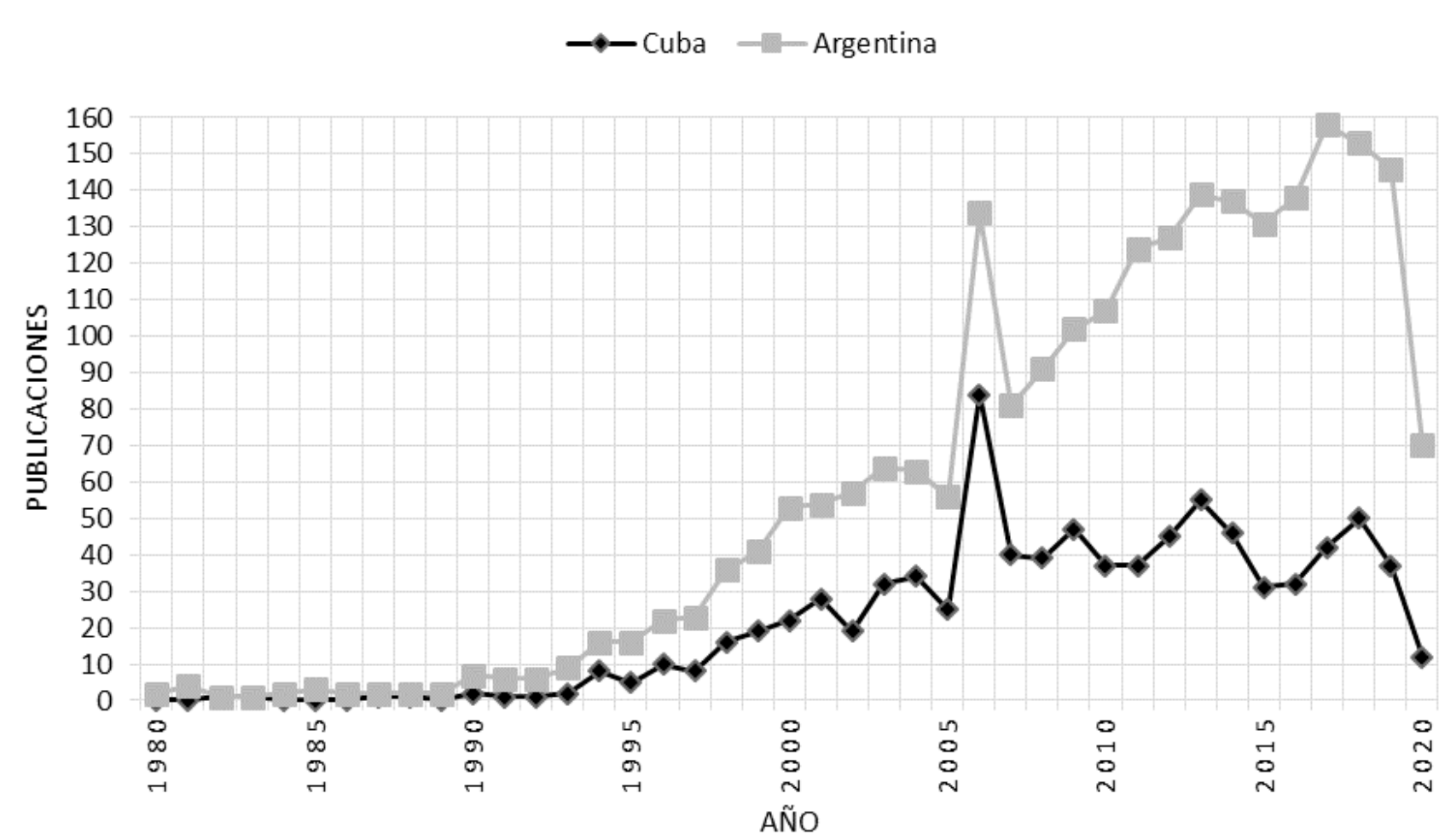

Figura 1. Cantidad de publicaciones por año sobre vacunas biotecnológicas, de Cuba y Argentina realizadas en el periodo 1980-2020. Fuente: elaboración propia con base en datos de la WoS.

el SARS-CoV-2, toma un lugar central dado que es indispensable para la creación de innovaciones que atiendan las necesidades sanitarias.

Para conocer la tendencia de la producción científica sobre vacunas biotecnológicas se realizó una búsqueda bibliométrica en la plataforma WoS . En la figura 1 se muestra que entre 1980 y 2020 se identificaron 870 publicaciones en Cuba, y 1.518 de Argentina. En ambos casos se observa un au- mento de la producción científica, año con año, lo cual es consistente con el aumento en todas las áreas del conocimiento debido a los cambios en el modo de producción de la CyT, derivado de las tecnologías digitales y la interconexión global. No obstante, como se puede ver en la Tabla 1, si se toman las publicaciones para diferentes años, el promedio de investigadores por publicación es mayor en Argentina.

\begin{tabular}{|c|c|c|c|c|c|c|}
\hline \multirow{2}{*}{ Año* } & $\begin{array}{c}\text { Número } \\
\text { de } \\
\text { autores } \\
\text { (A) }\end{array}$ & $\begin{array}{c}\text { Número de } \\
\text { publicaciones } \\
\text { (B) }\end{array}$ & $\begin{array}{c}\text { Promedio } \\
\text { (A/B) }\end{array}$ & $\begin{array}{c}\text { Número de } \\
\text { autores (C) }\end{array}$ & $\begin{array}{c}\text { Número de } \\
\text { publicaciones (D) }\end{array}$ & $\begin{array}{c}\text { Promedio } \\
\text { (C/D) }\end{array}$ \\
\hline $\mathbf{2 0 0 0}$ & 217 & 35 & 6.2 & 130 & 22 & 5.9 \\
\hline $\mathbf{2 0 0 6}$ & 412 & 53 & 7.8 & 700 & 84 & 8.3 \\
\hline $\mathbf{2 0 1 3}$ & 985 & 92 & 10.7 & 490 & 55 & 8.9 \\
\hline $\mathbf{2 0 1 7}$ & 1600 & 117 & 13.7 & 437 & 42 & 10.4 \\
\hline
\end{tabular}

Tabla 1. Promedio anual de investigadores por publicación sobre vacunas biotecnológicas en Cuba y Argentina. Fuente: elaboración propia con base en datos de la WoS.

*Los años se eligieron debido a que en esos periodos se observa un incremento de publicaciones. 


\begin{tabular}{|l|l|l|c|}
\hline $\begin{array}{l}\text { Instituciones que publican } \\
\text { trabajos sobre vacunas } \\
\text { biotecnológicas en Cuba }\end{array}$ & $\begin{array}{l}\text { Número de } \\
\text { publicaciones } \\
\text { en las que se } \\
\text { mencionan }\end{array}$ & $\begin{array}{l}\text { Instituciones que financian } \\
\text { investigaciones sobre vacunas } \\
\text { biotecnológicas en Cuba }\end{array}$ & $\begin{array}{l}\text { Número de } \\
\text { publicaciones } \\
\text { en las que se } \\
\text { mencionan }\end{array}$ \\
\hline CIGB & 354 & CIGB & 29 \\
\hline Instituto Finlay de Vacunas & 179 & $\begin{array}{l}\text { Programa Cubano para el } \\
\text { Desarrollo de la Vacuna contra el } \\
\text { Dengue }\end{array}$ & 19 \\
\hline Centro de Inmunología Molecular & 159 & Centro de Inmunología Molecular & 17 \\
\hline $\begin{array}{l}\text { Instituto de Medicina Tropical } \\
\text { Pedro Kourí }\end{array}$ & 144 & Instituto Finlay & 17 \\
\hline Universidad de la Habana & 59 & Organización Mundial de la Salud & 12 \\
\hline Organización Mundial de Salud & 42 & Gobierno Cubano & 9 \\
\hline Instituto Pasteur & 38 & Heber Biotec & 6 \\
\hline Hospital Hermanos Ameijeiras & 27 & Wellcome Trust & 6 \\
\hline $\begin{array}{l}\text { Hospital Celestino Hernández } \\
\text { Robau }\end{array}$ & 24 & $\begin{array}{l}\text { Centro Común de Investigación de } \\
\text { la Comisión Europea }\end{array}$ & 5 \\
\hline $\begin{array}{l}\text { Centro de Investigaciones } \\
\text { Médico-Quirúrgicas }\end{array}$ & 20 & $\begin{array}{l}\text { Consejo de Investigación Médica } \\
\text { Del Reino Unido }\end{array}$ & 5 \\
\hline
\end{tabular}

Tabla 2. Principales 10 instituciones que publican y financian investigaciones sobre vacunas biotecnológicas en Cuba (1980-2020). Fuente: elaboración propia con base en datos de la WoS.

Las instituciones que investigan sobre vacunas biotecnológicas en Cuba son promovidas y controladas por el Estado cubano. La Tabla 2 muestra a la izquierda las instituciones que realizan la mayor parte de las publicaciones cubanas sobre vacunas biotecnológicas y a la derecha las que financian mayoritariamente las investigaciones en este campo. En relación con estas últimas, se trata en su mayoría de instituciones públicas, lo que le permite al Estado cubano controlar las agendas de investigación y orientarlas en primera instancia a cubrir las necesidades sanitarias de la población y, en segundo lugar, a la creación de productos con los que puede competir en el mercado internacional.

La Tabla 3 muestra las instituciones más destacadas en la producción científica relacionada con vacunas biotecnológicas en Argentina. GlaxoSmithKline destaca como ente privado. EI MINCyT financia la mayor parte de los proyectos relacionados con vacunas biotecnológicas; también resaltan algunas instituciones gubernamentales estadounidenses y la Universidad de Buenos Aires. Mientras que las empresas transnacionales tienen poca participación en términos de investigación básica.

Se identificó que las publicaciones en conjunto no siempre obedecen a convenios celebrados entre instituciones o países, sino que surgen a partir de relaciones personales entre investigadores. Entre 1980 y 2020 Cuba colaboró con 87 países entre ellos EE.UU. (68 publicaciones); el Reino Unido (58), Argentina (38), Brasil (37), España (37) y México (24). En cuanto a Argentina, ha colaborado con 148 países entre ellos EE.UU. (396 publicaciones), Brasil (184), Francia (127), Alemania (119), España (108), Reino Unido (97) y México (93). Cabe mencionar que Argentina ha establecido convenios de colaboración con Brasil y Cuba y en menor medida con países europeos en el Horizonte 2020.

El análisis bibliométrico permitió observar que ambas naciones cuentan con una importante pro- 
| Ciencia, Tecnología y Política | Año 4 | Nº | e065 | Noviembre 2021 | ISSN 2618-3188 | www.revistas.un|p.edu.ar/CTyP |

\begin{tabular}{|c|c|c|c|}
\hline $\begin{array}{l}\text { Instituciones que } \\
\text { publican trabajos } \\
\text { sobre vacunas } \\
\text { biotecnológicas en } \\
\text { Argentina }\end{array}$ & $\begin{array}{l}\text { Número de } \\
\text { publicaciones } \\
\text { en las que se } \\
\text { mencionan }\end{array}$ & $\begin{array}{l}\text { Instituciones que } \\
\text { financian } \\
\text { Investigaciones sobre } \\
\text { vacunas } \\
\text { biotecnológicas en } \\
\text { Argentina }\end{array}$ & $\begin{array}{l}\text { Número de } \\
\text { publicaciones } \\
\text { en las que se } \\
\text { mencionan }\end{array}$ \\
\hline CONICET & 510 & MINCyT & 564 \\
\hline $\begin{array}{l}\text { Universidad de Buenos } \\
\text { Aires }\end{array}$ & 489 & $\begin{array}{l}\text { Departamento de Salud } \\
\text { y Servicios Humanos de } \\
\text { los Estados Unidos }\end{array}$ & 133 \\
\hline $\begin{array}{l}\text { Hospital de Niños "Dr. } \\
\text { Ricardo Gutiérrez" }\end{array}$ & 133 & $\begin{array}{l}\text { Institutos Nacionales de } \\
\text { Salud de Estados } \\
\text { Unidos }\end{array}$ & 128 \\
\hline $\begin{array}{l}\text { Universidad Nacional de } \\
\text { la Plata }\end{array}$ & 118 & $\begin{array}{l}\text { Universidad de Buenos } \\
\text { Aires }\end{array}$ & 114 \\
\hline Instituto Pasteur & 117 & $\begin{array}{l}\text { Instituto Nacional de } \\
\text { Alergias y } \\
\text { Enfermedades } \\
\text { Infecciosas de Estados } \\
\text { Unidos }\end{array}$ & 58 \\
\hline $\begin{array}{l}\text { Universidad Nacional de } \\
\text { Córdoba }\end{array}$ & 82 & GlaxoSmithKline & 56 \\
\hline Instituto Leloir & 78 & $\begin{array}{l}\text { Consejo Nacional de } \\
\text { Desenvolvimento } \\
\text { Científico e Tecnológico } \\
\text { de Brasil }\end{array}$ & 24 \\
\hline GlaxoSmithKline & 69 & Sanofi & 38 \\
\hline $\begin{array}{l}\text { Instituto Nacional de } \\
\text { Tecnología } \\
\text { Agropecuaria }\end{array}$ & 69 & $\begin{array}{l}\text { Fundación Bill y Melinda } \\
\text { Gates }\end{array}$ & 34 \\
\hline $\begin{array}{l}\text { Centros para la } \\
\text { Prevención del Control } \\
\text { de Enfermedades de } \\
\text { EE. UU. }\end{array}$ & 53 & Fundación Sales & 26 \\
\hline
\end{tabular}

Tabla 3. Principales 10 instituciones que publican y financian investigaciones sobre vacunas biotecnológicas en Argentina (1980-2020). Fuente: elaboración propia con base en datos de la WoS.

ducción académica en materia de vacunas biotecnológicas. Argentina dedica gran parte de sus esfuerzos a la creación de ciencia básica, presentando casi el doble de registros en comparación con Cuba. Sin embargo, no ocurre lo mismo en materia de patentamiento. La Figura 2 muestra las patentes concedidas a Cuba y Argentina referidas a productos farmacéuticos y biotecnológicos. Cuba sobresale en ambos casos.

En ambas naciones el financiamiento público en el área ha sido clave para impulsar el sector. No obstante, el nivel de desarrollo es distinto en cada país. A diferencia de Cuba, Argentina no ha logra- do vincular la I+D con la producción y comercialización, tampoco con la resolución de problemas en materia de salud, aunque cuenta con las capacidades tecnológicas necesarias para hacerlo.

\section{Conclusiones}

La ciencia, la tecnología y la innovación son una palanca para el desarrollo económico y social. Sin embargo, la inversión por sí sola no es suficiente para solucionar los problemas sociales, sino que tiene que ver la orientación de la política pública que se implementa desde el Estado. Tal como lo 


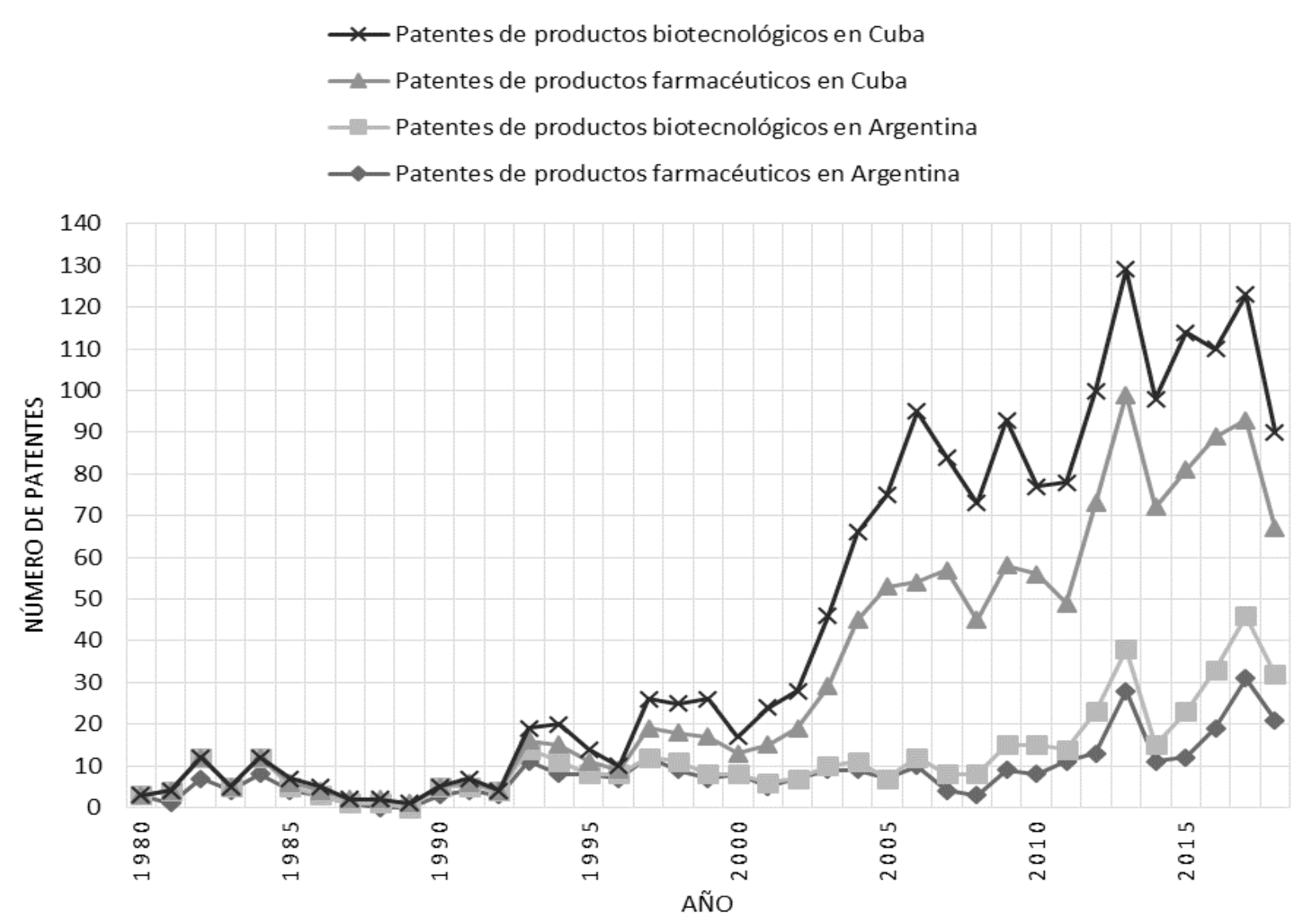

Figura 2. Patentes de productos farmacéuticos y biotecnológicos concedidos a Cuba y Argentina entre 1980 y 2018. Fuente: elaboración propia con datos de la Organización Mundial de Propiedad Intelectual [OMPI] (2020).

señala la Red PLACTS (2020), otro estilo tecnológico o de desarrollo es posible, pero es imperativo alejarse de las recetas dictadas por los países centrales, contar con más y mejores sinergias de cooperación sur-sur y, sobre todo, definir políticas desde el Estado, en donde se prioricen las necesidades de la población.

Si bien la biotecnología tiene la capacidad de incidir en la solución de problemas sanitarios, es necesario que el Estado no solo financie la I+D, sino que la articule con la producción, la comercialización y las necesidades de la población. Lo anterior, puede facilitar la socialización de las aplicaciones científico-tecnológicas y atender las necesidades del sistema de salud en cada país.

Cuba entendió tempranamente la necesidad de construir y promover una plataforma educativa y científica que sirviera para enfrentar los retos sanitarios de su población. La salud en este país se consideró como un área estratégica y durante varias décadas se impulsó la formación de personal altamente calificado, la construcción de infraestructura, el financiamiento a la I+D y la creación de empresas, entre otras. De este modo, el Estado ha instrumentado una política de ciclo cerrado que le permitió crear una plataforma científico -tecnológica virtuosa, que por un lado le posibilita cubrir las necesidades sanitarias de su población y por otro lado controlar el diseño, patentamiento y producción de vacunas.

Argentina ha desarrollado planes, programas y estrategias puntuales de impulso y financiamiento para el desarrollo de la biotecnología y de vacunas biotecnológicas que le han permitido conso- 
lidarse fuertemente en la ciencia básica asociada a este campo, lo que se refleja en la cantidad de publicaciones producidas en los últimos cuarenta años. Sin embargo, no ha logrado vincular la I+D con la producción y comercialización de los desarrollos y tampoco con la resolución de las necesidades y demandas sociales en materia de salud de su población.

\section{Bibliografía}

Albornoz, M. (2009). Desarrollo y políticas públicas en ciencia y tecnología en América Latina. Revista de Investigaciones Políticas y Sociológicas, 8(1), 65-75.

Anlló, G.; Añon, M. C.; Bassó, S.; Bellinzoni, R.; Bisang, R.; Cardillo, S.; Carricarte, V.; Cassullo, E.; Ciccia, G.; Corley, E.; Fuchs, M.; Genovesi, M.; Gutierrez, M. A.; Ortiz, I.; Pagano, E.; Plata, B.; Trigo, E.; Regunaga, M. (2016). Biotecnología argentina al año 2030: llave estratégica para un modelo de desarrollo tecno-productivo. Buenos Aires: Ministerio de Ciencia, Tecnología e Innovación Productiva - República Argentina. https://www.argentina.gob. ar/sites/default/files/est_bio_biotecnologia-argentina-al-2030-sintesis.pdf

BioCubaFarma (23 de junio de 2021). Quiénes somos. Recuperado el 23 de junio de 2021 de https://www.biocubafarma.cu/

Bolívar Zapata, F. G. (coord.) (2001). Biotecnología moderna para el desarrollo de México en el siglo XXI: retos y oportunidades. CONACYT y Fondo de Cultura Económica.

Cadalzo-Díaz, Y.; Caballero-Torres, I.; Becerra-Alonso, M. J. (2017). La gestión de capital humano en empresas del sector biotecnológico cubano. Ingeniería Industrial, 38(1), 18-31.

Decreto 335 de 2012 [Consejo de Ministros] Crea- ción de la Organización Superior de Dirección Grupo de las Industrias Biotecnológicas y Farmacéuticas sus funciones y facultades. Gaceta Oficial de la República de Cuba, 52(CX), 243-245.

Dirección General de Investigación e Innovación de la Comisión Europea (2014). HORIZON 2020 en breve. El Programa Marco de Investigación e Innovación de la Unión Europea. https://ec.europa. eu/programmes/horizon2020/sites/default/files/ H2O2O_ES_KIO213413ESN.pdf

Guzmán-Sánchez, M. V.; Piñón-Lora, M.; Villaseñor-García, E. A.; Jiménez-Andrade, J. L.; CarriIlo-Calvet, H. (2018). Characterization of the Cuban biopharmaceutical industry from collaborative networks. Scientometrics, 115(3), 1533-1548.

Ley 26270 de 2007. Promoción del Desarrollo y Producción de la Biotecnología Moderna. 25 de julio de 2007. República de Cuba

López, M. P. (2019). Cooperación en ciencia y tecnología entre Argentina y Cuba en el siglo XXI. El caso del Centro Argentino-Cubano de Biotecnología Aplicada al Desarrollo de Vacunas y Fármacos (2009-2015). Si Somos Americanos, 19(1), 139-164.

López, E. M.; Silva, R.; Acevedo, B.; Buxadó, J. A.; Aguilera, A.; Herrera, L. (2006). Biotechnology in Cuba: 20 years of scientific, social and economic progress. Journal of Commercial Biotechnology, 13(1), 1-11.

Ministerio de Ciencia, Tecnología e Innovación Productiva - República Argentina [MINCyT] (diciembre-marzo 2010). Boletín Estadístico Tecnológico. Biotecnología, 4.

Ministerio de Ciencia, Tecnología e Innovación Productiva - República Argentina [MINCyT] (Noviembre de 2016). Las empresas de biotecnología en Argentina. Documento de trabajo. https://www.argentina.gob.ar/sites/default/files/ est_bio_las-empresas-de-biotecnologia-en-ar- 
gentina-2016.pdf

Ministerio de Ciencia, Tecnología e Innovación Productiva - República Argentina y Secretaría de Planeamiento y Políticas en Ciencia, Tecnología e Innovación Productiva [MINCyT y SEPP]. (s.f.). Argentina Innovadora 2020. Plan Nacional de Ciencia, Tecnología e Innovación. Lineamientos estratégicos 2012-2015. https://www.argentina.gob.ar/ sites/default/files/pai2020.pdf

Ministerio de Salud - República Argentina (16 de junio de 2021). ANLIS MALBRÁN. Misión, Visión y Objetivos. Recuperado el 16 de junio de 2021 de http://www.anlis.gov.ar/institucional/

Organización Mundial de la Propiedad Intelectual [OMPI] (15 de enero de 2020). Patentes concedidas, por sector de la tecnología-Recuento total de oficina de presentación [Excel]. Centro de datos estadísticos de la OMPI sobre propiedad intelectual. https://www3.wipo.int/ipstats/index.htm?lan$g=e s$

Organización Panamericana de la Salud [OPS] (17 de enero de 2020). Biotecnología para la salud en Cuba. Información general. Recuperado el 17 de enero de 2020 de https://www3.paho.org/cub/ index.php?option=com_content\&view=article\&i$d=211$ :biotecnologia-salud-cuba\&ltemid $=273$

Plahte, J. (2010). Strategic Evaluations and Techno-Economic Networks. Vaccine In- novation in the Cuban Biotech Sector: for Public Health-or for Profits?, Working Papers on Innovation Studies, 20100108. https://ideas.repec.org/p/tik/inowpp/20100108.html

Quach, U.; Thorsteinsdóttir, H.; Renihan, J.; Bhatt, A.; von Aesch, Z. C.; Daar, A. S.; Singer, P. A. (2006). Biotechnology Patenting Takes Off in Developing Countries, International Journal of Biotechnology, 8, 43-59.

Red de Pensamiento Latinoamericano en Ciencia,
Tecnología y Sociedad [Red PLACTS] (2020). Otro estilo científico y tecnológico es posible. Ciencia, tecnología y política, 3(5), 050. https://doi.or$\mathrm{g} / 10.24215 / 26183188 \mathrm{e} 050$

Reid-Henry, S.; Plahte, J. (2020). ¿Inmunidad ante Ios ADPIC? La producción de vacunas y la industria biotecnológica en Cuba. Revista Bienestar, 1(1) 218-237.

Varsavsky, O. (1978). Estilos tecnológicos. Propuestas para la selección de tecnologías bajo racionalidad socialista. Ediciones Periferia S.R.L.

Vitagliano, J. C.; Villalpando, F. A. (2003). Análisis de la biotecnología en Argentina. Programa de Fortalecimiento Institucional de la Política Comercial Externa. Préstamo BID 1206/OC-AR. Diseño de Programas Piloto Sectoriales de Exportación. http://www.foarbi.org.ar/docs/BiotecArgV1.pdf 